\title{
Cord Blood Direct Antiglobulin Test (DAT) Positivity in RhD Incompatible Neonates-A Two Years Study
}

\author{
S. S. S. Quadri ${ }^{1}$, Srilatha Bollipogu ${ }^{2^{*}}$, S. Srujana ${ }^{3}$, Naval Kishore ${ }^{4}$ \\ ${ }^{1}$ Associate Professor, Department of Pathology, Government Medical College, Mahabubnagar, Telangana, India \\ ${ }^{2}$ Assistant Professor, Department of Pathology, Government Medical College, Mahabubnagar, Telangana, India \\ ${ }^{3}$ Associate Professor, Department of Pathology, Government Medical College, Mahabubnagar, Telangana, India \\ ${ }^{4}$ Professor \& HOD, Department of Pathology, Government Medical College, Mahabubnagar, Telangana, India
}

*Address for Correspondence: Dr. Srilatha Bollipogu, Assistant Professor, Department of Pathology, Government Medical College, Mahabubnagar, Telangana, India

E-mail: drsrilathajkp@yahoo.com

Received: 20 Sep 2020/ Revised: 08 Nov 2020/ Accepted: 12 Feb 2021

\begin{abstract}
Background: Rh D negative blood in mothers carrying Rh D positive fetus is usually associated with the hemolytic disease of the newborn. Because affected neonates may develop severe hyperbilirubinemia, early identification of at-risk neonates is critically important. We hypothesized that the uses of the direct antibody test (DAT) help us in identifying those neonates who meet the treatment criteria for hyperbilirubinemia. This study aimed to review the incidence of positive DAT in cord blood samples at Government General Hospital, Mahabubnagar, Telangana, India.

Methods: We retrospectively reviewed all results of Direct Anti Globulin (DAT) for two years from January 2019 to December 2020, which was routinely performed in cord blood samples. Direct Anti Globulin (DAT) test was performed on 854 cord blood samples using standard procedures.

Results: $29 / 854(3.39 \%)$ of the cord blood samples were DAT positive. In this DAT positive was seen in 13/29 (44.82\%) of O positive blood group; $7 / 29(24.13 \%)$ of A positive blood group; $9 / 29(31.03 \%)$ of B positive blood group and we did not encounter any DAT positive cases in $A B$ positive blood group.

Conclusion: Due to DAT positivity in cord blood, for the benefit and protection of the neonates and mother in India, there is a need to establish intervention programmes in terms of neonatal screening and immunoprophylaxis.
\end{abstract}

Key-words: Cord blood, Direct antiglobulin test, Hemolytic disease of the newborn, Hyperbilirubinemia, Rh-negative mothers

\section{INTRODUCTION}

The direct antiglobulin test (DAT), also known as the Direct Coombs' test, is a screen for non-agglutinating antibodies present on an individual's red cells. The principle of the test was described by Moreschi in 1908, and it was rediscovered by Coombs et al. ${ }^{[1]} 1945$. When a pregnant woman's serum contains a clinically significant immunoglobulin $\mathrm{G}$ ( $\operatorname{lgG}$ ) antibody directed against an antigen present on the red cells of her unborn child, placental transfer of the antibody into the fetal circulation may destroy fetal red cells resulting in Hemolytic Disease of the Newborn (HDN).

\section{How to cite this article}

Quadri SSS, Bollipogu S, Srujana S, Kishore N. Cord Blood Direct Antiglobulin Test (DAT) Positivity in RhD Incompatible Neonates-A Two Years Study. SSR Inst. Int. J. Life Sci., 2021; 7(2): 2774-2780.

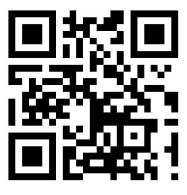

Access this article online https://iijls.com/
At its worst, the anaemia may result in hydrops fetalisgross oedema and heart failure leading to fetal death.

Neonatal hyperbilirubinemia places the infant at risk of neurological damage. Phototherapy converts bilirubin to an excretable photoproduct. ${ }^{[1,2]}$ The indications for phototherapy and exchange transfusion are determined by the serum bilirubin, age/gestation and bodyweight.

The Rhesus negative group refers to the genotype in which the three major genes (CDE) are absent. Thus, the genotype of the Rhesus negative individual is ' $C D E$ ' ${ }^{[2]}$. Sensitization of Rh-negative mother occurs during childbirth when the mother's bloodstream penetrates a certain amount of Rh-positive child erythrocytes. Erythrocytes, as a foreign substance to the mother, encourage her body to begin to produce Rh antibodies. Therefore, the second and other pregnancy can have complications related to maternal $\mathrm{Rh}$ antibodies to $\mathrm{Rh}$ positive fetal red blood cells. Their appearance in the circulation is also possible after amniocentesis, 
spontaneous or induced abortion, cordocentesis, chorionic villus sampling, ruptured ectopic pregnancy and blunt trauma to the abdomen ${ }^{[3,4]}$. An Rh-negative mother after sensitized produces anti-Rh IgG antibodies that cross the placenta and the risk factors for antibody production has been reported to include second and later pregnancies, feto-maternal incompatibility in the $A B O$ system, paternal zygosity, maternal toxaemia and antigen load. ${ }^{[5]} \quad$ Direct antiglobulin test (DAT) determination allows us to identify the presence of anti erythrocyte antibodies of IgG isotope, originating in maternal serum on the surface of the erythrocytes of the fetus or newborn. ${ }^{\text {6-8] }}$

Recommendations by The British Committee for Standardization in Haematology (BCSH) says that infant born to Rh $D$ negative mothers should have umbilical cord blood $A B O$ and Rh tested ${ }^{[9,10]}$. If the baby is Rh $D$ positive, maternal samples should then be taken for assessment of feto-maternal haemorrhage so that adequate anti-RhD immunoprophylaxis can be given to the mother. The American Academy of Pediatrics (AAP) revised its recommendations regarding the identification and management of neonatal jaundice in 2004. In those guidelines, the AAP recommends "all pregnant women should be tested for ABO and Rh-D blood types and have a serum screen for unusual isoimmune antibodies." The definition of meeting the treatment criteria for hyperbilirubinemia was attained if any of the three scenarios occurred during the 1st week of life: (1) Neonate met the criteria for phototherapy based on AAP guidelines by having any Total Serum Bilirubin (TsB) level above threshold for phototherapy based on age since birth, gestational age, and presence of neurotoxicity risk factors; (2) Received intravenous immunoglobulin (IVIG) for what was deemed severe HDN by the attending physician; (3) Received a double volume exchange transfusion. ${ }^{[11]}$

Determination of blood type in $A B O$ Rh $D$ negative pregnant women allows reasonable precautions which limit the risk to the fetus. Erythroblastosis is a very serious medical condition for about 4000 babies a year. In $15 \%$ of cases of babies die before birth. Those who survive may suffer from jaundice, which leads to deafmuteness, speech disturbances, cerebral palsy and mental retardation. The use of the direct antiglobulin test (DAT) would be of high predictive value in identifying those neonates most likely to meet treatment criteria for hyperbilirubinemia. ${ }^{[3,4]}$

\section{MATERIALS AND METHODS}

Data Collection- Data of women visiting for antenatal care at Government General Hospital, Mahabubnagar, from January 2019 to December 2020, for deliveries were collected from records. Blood group details of the patients visited for antenatal care during each month of the year were noted down. The number of candidates belonging to the $A, B, A B$ and $O$ groups was consolidated. Rh factor details of the patients were also consolidated.

Inclusion Criteria- A total of 895 healthy Rhesus negative pregnant mothers, who attended the antenatal clinic were included in the study. We retrospectively reviewed all results of DAT, which was routinely performed in cord blood samples of all infants born to Rh-negative mothers between January 2019 to December 2020. During this period, a total of 854 cord blood samples were analyzed.

Exclusion Criteria- Rh positive pregnant mothers, who attended the antenatal clinic between January 2019 to December 2020 were excluded from the study.

Slide technique- Hemagglutination was used for the determination of $A B O$ blood grouping. For the direct phase, commercial reagents were used that contained monoclonal antibodies with anti-A, anti-B and anti-AB specificity (Tulip Diagnostics private limited).

A drop of blood from each volunteer was placed on a glass slide in three places. A drop of each of the antisera A, B and D was added and mixed with each blood sample, with the aid of glass rods. Monoclonal blood grouping antibodies, the in-vitro diagnostic reagent of Tulip Diagnostics private limited are used. Then, the mixture was rocked gently for 60 seconds to observe for agglutination. The results of agglutination were recorded immediately after mixing. The agglutination in blood drop $A$ was considered as group $A$ and agglutination in blood drop $B$ as group $B$. The agglutination in both drops was considered as group $A B$, and if both blood drops were not agglutinated, it was considered as group $O$. The agglutination in rhesus blood drop was considered as rhesus positive and non-agglutination as rhesus negative. ${ }^{[12]}$

After delivery of newborn DAT was performed by using whole blood samples obtained by a puncture of the umbilical vessel at the end of the placenta immediately. 
The umbilical cord was clamped at the end attached to the neonate. Another cord clamp was placed 8 to 10 inches away from the first. The isolated section was cut and the fetal blood sample was collected into an ethylene diamine tetraacetic acid (EDTA) container. The bottle was labelled with patient identity including name and hospital number. Immediately at $4000 \mathrm{rpm}$ for 2-3 minutes the umbilical cord blood was centrifuged to enable the red cells to sediment. Supernatant plasma was removed leaving the fetal red cells in the tube.

Direct Antiglobulin Test (DAT) on cord blood was performed by taking two tubes $(12 \times 75 \mathrm{~mm})$ which were labelled 'test' and 'control' and a drop of a $3 \%$ suspension of cord blood added to them. The cells in the tube were washed three times with saline and the saline was completely decanted after the last wash. To the test tube labelled 'test' was added 2 drops of Anti Human Globulin (AHG) reagent (Monoclonal anti-lgG Tulip Diagnostics private limited) while 2 drops of $3 \%$ of bovine albumin (Tulip Diagnostics private limited) was added to the tube labelled 'control'. Both tubes were centrifuged at $3000 \mathrm{rpm}$ for 15 to 20 seconds. Following centrifugation, the cell pellet was completely resuspended by gentle tipping and rolling of the tube. The macroscopical examination was done and agglutination was scored. In doubtful cases, the presence or absence of agglutination was confirmed with low power magnification. For control, to all negative tubes was added 1 drop of control cells weakly sensitized with IgG, it was mixed, centrifuged and observed for agglutination. A mixed field weakly positive reaction at this stage indicates that the AHG had been added to the tube and it was still reactive. All reactive results at this stage were considered valid. The rate of alloimmunization in vivo among Rh $\mathrm{D}$ negative mothers has not been determined.

Statistical Analysis- The prevalence in this study was determined from the proposition of individuals and the total study population and is expressed as a percentage. Descriptive statistics of variable and other characteristics of the sampled population were calculated and tabulated are expressed as percentages. All the statistical analysis was done using the Statistical Package for Social Sciences (SPSS) software package version 20.

Ethical Approval- Ethical review and clearance were obtained from the Hospital and Ethics Committee.
Department protocol was via informed written consent before data collection.

\section{RESULTS}

We found that a total of 20863 deliveries were conducted from January 2019 to December 2020. We evaluated the ABO blood groups of 895/20863 (4.58\%) Rhesus $D$ negative pregnant mothers in two years study and out of 895 deliveries, 854 (95.41\%) were Rh positive babies born to Rh-negative mothers and $41(4.58 \%)$ were Rh negative babies born to Rh-negative mothers as shown in Fig. 1 and were excluded from the study. DAT positive cases were seen in 29/854 (3.39\%) of cord blood samples and DAT negative cases are seen in 825/854 (96.6\%) in Government General Hospital, Mahabubnagar as shows in Fig. 2.

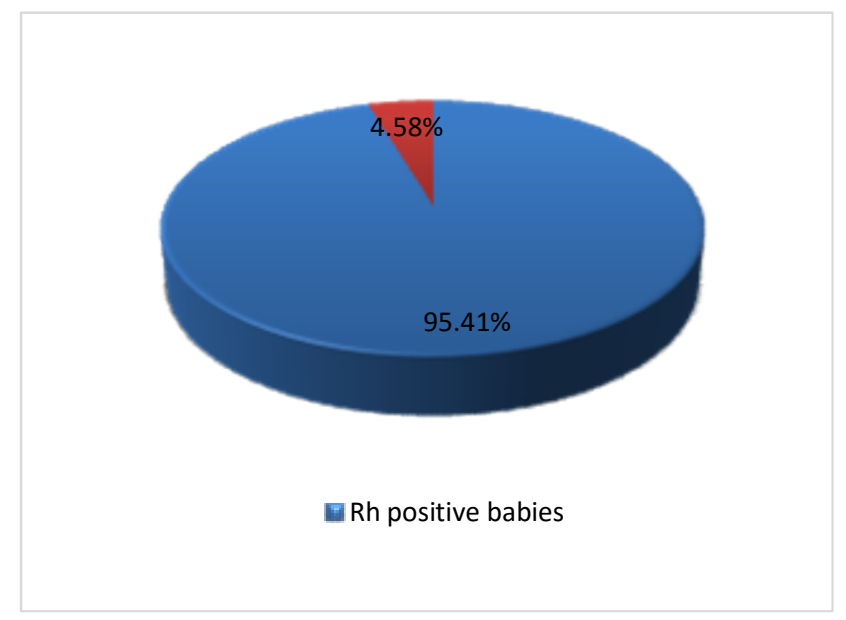

Fig. 1: Distribution of total Rh positive and Rh negative babies born in Government General Hospital, Mahabubnagar, from January 2019 to December 2020

Of 854 Rh-positive babies 303/854 (35.48\%) babies are of blood group O, 216/854 (25.29\%) group A, 254/854 (29.74\%) group $B$ and $81 / 854$ (9.48\%) group $A B$ as shown in Fig. 3. In this DAT positive was seen in 13/29(44.82\%) of $O$ positive blood group; $7 / 29(24.13 \%)$ of $A$ positive blood group; 29(31.03\%) of B positive blood group and we did not encounter any DAT positive cases in $A B$ positive blood group as shown in Fig. 4 . In this study, it is shown that majority of DAT positive cases were seen in the $O$ positive blood group, which is shown in Table 1. 


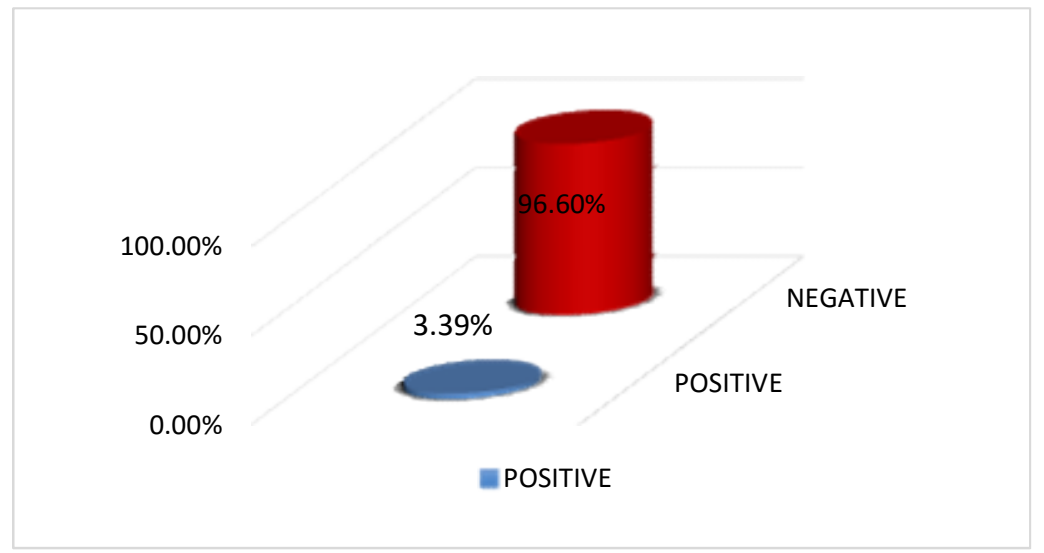

Fig. 2: Relative percentage of DAT positive and negative cases at Government General Hospital, Mahabubnagar

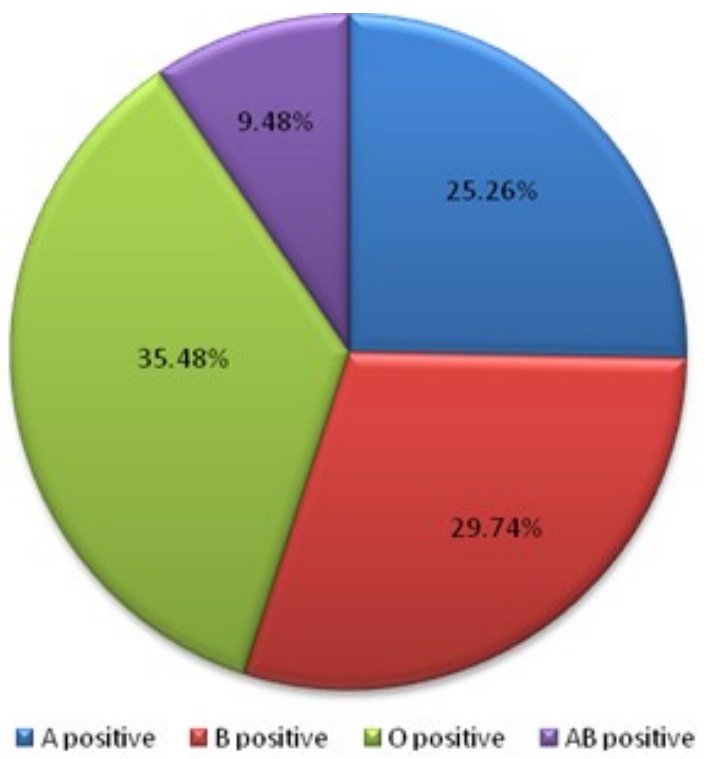

Fig. 3: Total distribution of blood groups in positive blood group babies born in Government General Hospital, Mahabubnagar from January 2019 to December 2020

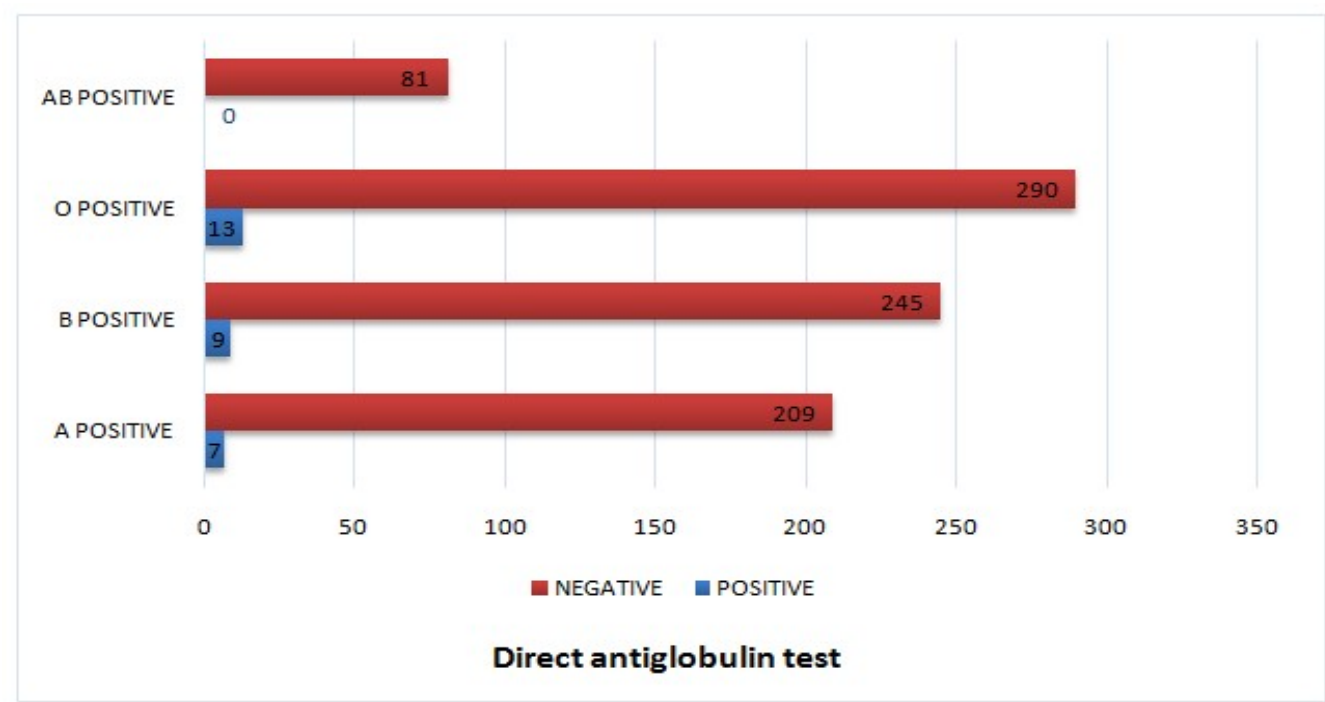

Fig. 4: Relative distribution of positive and negative Direct Antiglobulin Test (DAT) in individual blood group 
Table 1: Prevalence of Positive DAT among Rh-Positive Babies Born to Rh Negative mothers in Government General Hospital, Mahabubnagar

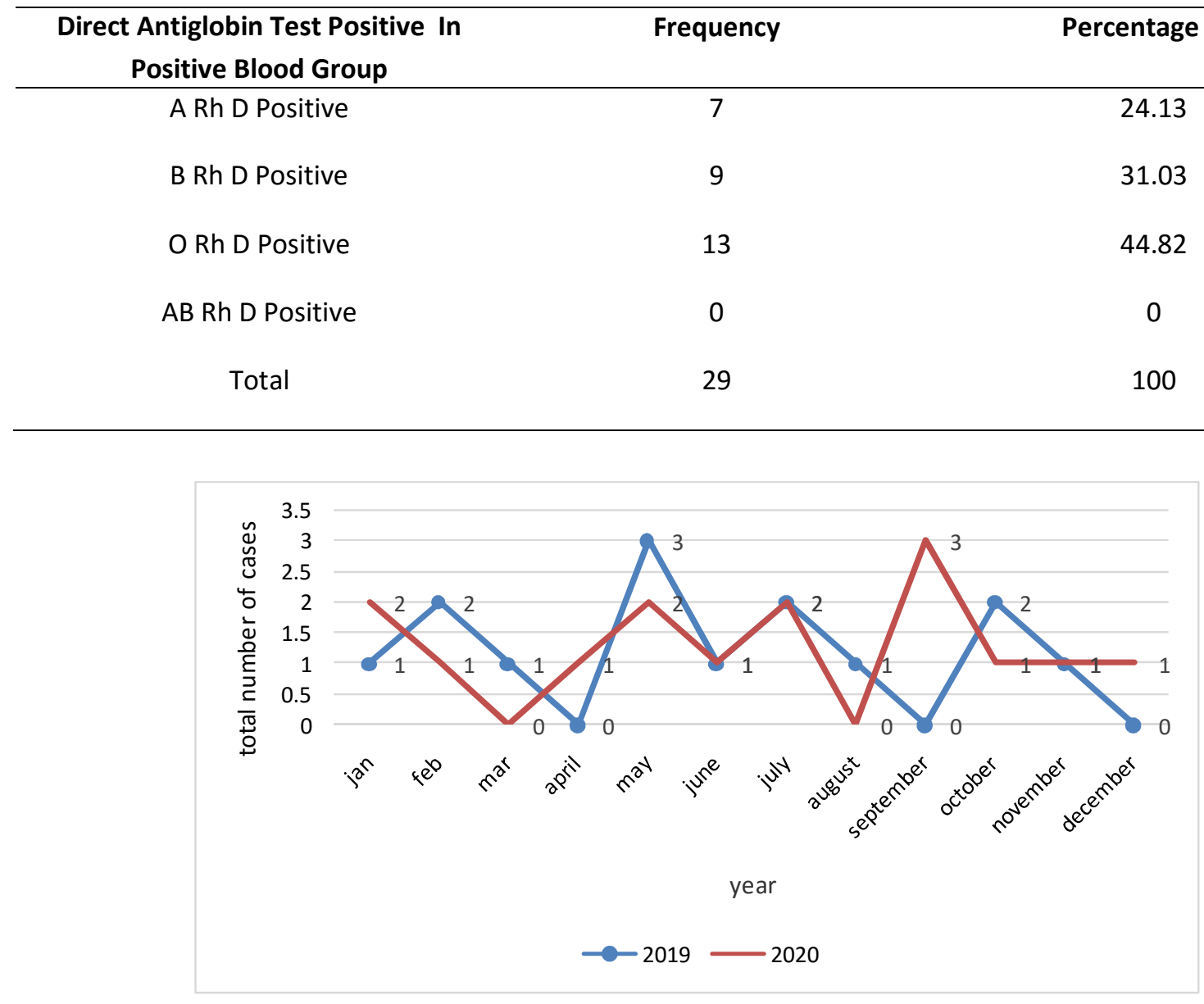

Fig. 5: Distribution of the total number of direct antiglobulin test positive cases monthly wise in the years 2019 and 2020

Table 2: Distribution of DAT in Cord blood samples of Rh Positive Babies Born to Rh Negative mothers in Government General Hospital, Mahabubnagar

\begin{tabular}{ccc}
\hline Direct antiglobulin test & Frequency & Percentage (\%) \\
\hline Negative & 825 & 96.60 \\
Positive & 29 & 3.39 \\
Total & 854 & 100
\end{tabular}

\section{DISCUSSION}

The knowledge of the blood groups and Rhesus factor is important in evolution, related to diseases and environment, essential in blood transfusion, organ transplantation, forensic pathology, anthropology and training ancestral relation of human, and also helps to prevent complications due to Rhesus incompatibility. ${ }^{[13-}$ ${ }^{15]}$ The prevalence of Rh D negative women in this study was $4.58 \%$. This is similar to previous studies done at Ibadan and Abraka in Nigeria. ${ }^{[16,17]}$ This rate shows a low frequency of $R h D$ negative blood group system in this environment. This finding is similar to that previously reported amongst African subjects, West Indians, and blacks in Great Britain ${ }^{[18] .}$ The results are, however, different from those reported from the Eastern highlands of Papua Guinea where the entire population was 
reported to be $100 \% \mathrm{Rh}$ D positive. ${ }^{[19]}$ DAT is a useful tool in the early prediction of jaundice or hyperbilirubinemia. In our study, $3.39 \%$ of the prevalence rate of positive DAT was seen which is shown in Table 2.

$1-9 \%$ of prevalence rate was reported by Dinesh ${ }^{[1]}$ and Cianciarullo ${ }^{[18]}$ in White populations. The incidence of a positive DAT in newborns was 3.39\%, a percentage higher than that (2.3\%) reported by Dillon et al. ${ }^{[24]}$ but lower than that $(3.5 \%)$ of Hershel et al. ${ }^{[20]}$ Higher the prevalence rate of positive cord blood DAT places women at higher risk of developing the hemolytic disease of the newborn. $23 \%$ of infants with positive DAT require phototherapy was reported by one study. ${ }^{[20]}$ In another study of the infants receiving phototherapy, $15.1 \%$ show a DAT positive ${ }^{[21]}$. Detection of positive DAT on cord blood enables the newborn at risk of clinically relevant jaundice to benefit from programs aimed at identifying risk or early detection of various adverse clinical conditions such as hyperbilirubinemia, encephalopathy, prolonged hospitalization, intense hemolytic anaemia or others. By inclusion of DAT in neonatal screening allows the early intervention with prophylactic phototherapy ${ }^{[22]}$.

In vivo phenomenon associated with a positive DAT could be due to several factors such as transfusion, druginduced and autoimmune hemolytic anaemia ${ }^{[23]}$. For women with a high percentage having received a previous blood transfusion and with multiple pregnancies, it is possible that alloantibodies in the recipient $R h D$ negative mothers must have sensitized their red cells to cause in vivo sensitization.

DAT results in our study showed a promising ability to predict the treatment criteria for hyperbilirubinemia in high-risk neonates. Positive DAT result close to birth may alert the clinician to apply more aggressive management techniques (e.g., frequent laboratory draws, prophylactic phototherapy, etc.), while a negative result may allow clinicians to adopt a more conservative approach and spare the neonate from overaggressive laboratory investigations or other invasive modalities. Early riskstratification using DAT results obtained early, can be one of the many tools available to clinicians in identifying and efficiently managing patients at risk for severe hyperbilirubinemia.

\section{CONCLUSIONS}

DAT being a useful tool in the early prediction of hyperbilirubinemia and if included as a neonatal screening test will help in identifying at-risk neonates. Maternal screening tests and a careful look at the history of drug administration during pregnancy could identify other important but rare causes of DAT positivity. Inclusion of DAT in neonatal screening allows early and immediate intervention with prophylactic phototherapy of at-risk neonates.

The use of universal DAT testing along with maternal isoimmunization will lead to further investigation and discussion to determine its efficacy and costeffectiveness in predicting severe hyperbilirubinemia.

\section{CONTRIBUTION OF AUTHORS}

Research concept- Dr. Srilatha Bollipogu

Research design- Dr. S.S.S.Quadri

Supervision- Dr. S.S.S.Quadri

Materials- Dr. Srilatha Bollipogu

Data collection- Dr. Srilatha Bollipogu

Data analysis and Interpretation- Dr. Srilatha Bollipogu

Literature search- Dr. S. Srujana

Writing article- Dr. S. Srujana

Critical review- Dr.S. S.S.Quadri

Article editing- Dr.S. S.S.Quadri

Final approval- Dr. Naval Kishore

\section{REFERENCES}

[1] Dorothy D. Review of positive direct antiglobulin tests found cord blood sampling. J Paediatr Child Health, 2005; 41(9-10): 504-07.

[2] Mollison PL, Engelfriet CP, Contreras M. Blood Transfusion in Clinical Medicine. (10thedn), Oxford, UK: Blackwell Sci., 1993; 543-91.

[3] Sebija I. Occurrence of $A B O$ and RhD Incompatibility with Rh Negative Mothers, Mater Sociomed. 2013; 25(4): 255-58. doi: 10.5455/msm.2013.25.255-258.

[4] Phibbs, Roderic H. Hemolytic Disease of the Newborn (Erythroblastosis Fetalis). In: Rudolph's Pedriatic, ed. Abraham, M. Rudolph, et al. Stamford: Appleton \& Lange, 1996.

[5] James RM, McGuire W, Smith DP. The investigation of infants with RhD negative mothers, can we safely omit the umbilical cord blood direct antiglobulin test. Arch Dis Child Fetal Neonatal Ed., 2011; 96: F301304.

[6] Dittmar K, Procter JL, Cipolone K, Njoroge JM, Miller $J$, et al. Comparison of DATs using traditional tube agglutination to gel column and affinity column procedures. Transfusion, 2001: 41: 1258-62. 
[7] Novaretti MC, Jens E, Pagliarini T, Bonifacio SL, Dorlhiac-Llacer PE, et al. Comparison of conventional tube test technique and gel microcolumn assay for direct antiglobulin test: a large study. J Clin Lab Anal., 2004; 18: 255-58. doi: 10.1002/jcla.20033.

[8] Meberg A, Johansen KB. Screening for neonatal hyperbilirubinaemia and $A B O$ alloimmunization at the time of testing for phenylketonuria and congenital hypothyreosis. Acta Paediatr., 1998; 87: 1269-74.

[9] Jeremiah ZA, Pwana FE, Mgbere O. Positive Cord Blood Direct Anti Globulin Test (DAT) is Strongly Associated with Parity and Maternal Age among Rh Negative Mothers in Maiduguri, Nigeria. J Blood Disord Transfus., 2013; S10: 002. doi: 10.4172/21559864.S10-002.

[10]Gooch A, Parker J, Wray J, Qureshi H. British Committee for Standards in Haematology Blood Transfusion Task Force, Guideline for blood grouping and antibody testing in pregnancy. Transfus Med., 2007; 17: 252-62. doi: 10.1111/j.1365-3148.2007. 00767.x.

[11]American Academy of Pediatrics Subcommittee on Hyper bilirubinemia. Management of hyper bilirubinemia in the newborn infant 35 or more weeks of gestation. Pediatr., 2004; 114(1): 297-316.

[12]Sultana R, Yousuf R, Rahman Z, Helali AM, Mustafa S, et al. Study of $A B O$ and RH-D Blood Groups among the common people of capital city of Bangladesh. Int J Pharm Pharm Sci., 2013; 5(3): 814-16.

[13]Khurshid B, Naz M, Hassan M, Mabood SF. Frequency of $A B O$ and Rh (D) blood groups in district Swabi, NWFP, Pakistan. J. Sci. Tech. Univ. Peshawar, 1992; 16: 5-6.

[14]Bamidele O, Arokoyo DS, Akinbola AO. Distribution of $A B O$ and rhesus blood groups among medical students in Bowen University, Iwo, Nigeria. Ann Biol Res., 2013; 4 (11): 1-6.

[15]Zaman et al. Study of $A B O$ and Rh-D blood group among the common people of Chittagong city corporation area of Bangladesh. I Public Health Epidemiol., 2015; 7(9): 305-10.
[16]Onwukeme KE. Blood group distribution in blood donors in Nigeria population. Niger J Physiol Sci., 1990; 6: 67- 70. doi: 10.23937/2377-9004/1410034.

[17]Kotila TR, Odukogbe AA, Okunola MA, Olayemi O, Obisesan KA. The pregnant Rhesus negative Nigerian women. Nigerian J Clinical Practice, 2005; 15: 4.

[18]Cianciarullo MA, Ceccon ME, Vaz Fv. Prevalence of immunohematologic tests at birth and the incidence of hemolytic disease in the newborn. Rev Assoc Med Bras., 2003; 49(1): pp. 45-53. doi: 10.1590/S010442302003000100033.

[19]Prevalence of rhesus negativity among pregnant women, International Journal of Research in Medical Sciences Nagamuthu EA et al. Int J Res Med Sci., 2016; 4(8): 3305-09. doi: http://dx.doi.org/10. 18203/2320-6012.ijrms20162284.

[20]Herschel M, Karrison T, Wen M, Caldarelli L, Baron B. Isoimmunization is unlikely to be the cause of hemolysis in $\mathrm{ABO}$-incompatible but direct antiglobulin test-negativeneonates. Pediatrics, 2002; 110(1): 127-30. doi: 10.1542/peds.110.1.127.

[21]Kaplan M, Kaplan E, Hammerman C, Algur N, Bromiker $\mathrm{R}$, et al. Post-phototherapy neonatal bilirubin rebound: a potential cause of significant hyperbilirubinaemia. Arch Dis Child, 2006; 91: 31-34.

[22]Adeniji AA, Fuller I, Dale T, Lindow SW. Should we continue screening rhesus $D$ positive women for the development of atypical antibodies in late pregnancy? J Matern Fetal Neonatal Med., 2007; 20: Pages 59-61. doi: 10.1080/14767050601123317.

[23]Baptista-Gonzaliz H, Hemandez-Martinez A, GalindoDelgado P, SantamariaHernandez C, RosenfieldMann F. Usefulness of direct antiglobulin test in neonatal screening. Bol Med Hosp Infant Max., 2009; 66: 502-10.

[24]Dillon A, Chaudhari T, Crispin P, Shadbolt B, Kent A. Has anti-D prophylaxis increased the rate of positive direct antiglobulin test results and can the direct antiglobulin test predict need for phototherapy in Rh/ABO incompatibility? J Paediatr Child Health, 2011; 47(1-2): 40-43. doi: 10.1111/j.1440-1754.2010. 01888.x. 